\title{
« Je ne vous opérerai pas. »
}

Registres argumentaires des refus d'opérer en chirurgie esthétique

The Main Justifications Put Forward by Cosmetic Surgeons When Rejecting Clients

\section{Yannick Le Hénaff}

\section{(2) OpenEdition \\ Journals}

Édition électronique

URL : http://journals.openedition.org/travailemploi/6748

DOI : 10.4000/travailemploi.6748

ISSN : $1775-416 \mathrm{X}$

\section{Éditeur}

DARES - Ministère du Travail

\section{Édition imprimée}

Date de publication : 1 octobre 2015

Pagination : 61-80

ISSN : 0224-4365

\section{Référence électronique}

Yannick Le Hénaff, « « Je ne vous opérerai pas. » », Travail et Emploi [En ligne], 144 | octobre-décembre 2015, mis en ligne le 01 octobre 2017, consulté le 20 avril 2019. URL : http://journals.openedition.org/ travailemploi/6748; DOI : 10.4000/travailemploi.6748 


\title{
« Je ne vous opérerai pas. »
}

\author{
Registres argumentaires \\ des refus d'opérer en chirurgie esthétique
}

\author{
Yannick Le Hénaff
}

\begin{abstract}
Dans cet article, nous nous sommes intéressé au cas original des refus d'opérer en chirurgie esthétique, en nous basant sur quatre-vingts entretiens avec des praticiens, des observations en congrès et une analyse de revues professionnelles. Écartant les réserves anatomiques ou physiologiques, les chirurgiens enquêtés expliquent leur décision par l'inadéquation entre le comportement ou la demande du client et leurs propres attentes. Le demandeur est alors rangé dans la catégorie de client à problèmes, en raison des conflits potentiels, voire des ennuis judiciaires, qu'il pourrait générer. Différents niveaux de lecture ont été proposés qui ont pour dénominateur commun le recours aux notions d'autonomie et d'identité professionnelles des chirurgiens, qui permet notamment de mettre en évidence leur fragilité médicale. Par ailleurs, sous couvert de déontologie (en se désintéressant des bénéfices financiers d'une opération), ces professionnels s'autorisent à écarter les clients qu'ils jugent indignes ou potentiellement dangereux, et ouvrent donc la porte à des processus de discriminations.
\end{abstract}

$\mathrm{L}$ es travaux sociologiques portant sur la décision médicale ont largement interprété le fait de prescrire comme une preuve de l'action. La prescription est considérée sous deux angles : comme le signe d'une culture professionnelle ou comme la démonstration de la pression des clients ${ }^{1}$ pour ne pas « rentrer les mains vides » (BROOM et al., 2014, p. 55, nous traduisons ; STIVERS, 2002). Dans cet article portant

\footnotetext{
* Laboratoire DySoLa (Dynamiques sociales et langagières), université de Rouen ; yannick.lehenaff@yahoo.fr.

1. En faisant référence au «client » et non au «patient», il ne s'agit surtout pas d'affirmer que le client est «le demandeur et le juge de toute production et de toute activité » (BROUSSARD et al., 2006, p. 210), mais de développer une posture en rupture avec les rhétoriques des professions « prestigieuses ». Le terme de patient est pétri de jugements de valeurs, et constitue une description idéalisée non seulement de l'individu en attente de réponse médicale, mais aussi de la relation avec le médecin. En analysant l'organisation de l'activité médicale - et non seulement la chirurgie esthétique - comme n'importe quelle autre relation de service, avec ses spécificités, mettant aux prises un professionnel et un client dans un environnement concurrentiel, on s'autorise les comparaisons irrévérencieuses (HUGHES, 1996).
} 
sur la chirurgie esthétique, ce sont au contraire les refus qui ont retenu notre attention. Plus précisément, nous nous intéressons à des cas de refus dans lesquels la disgrâce physique est reconnue et pour laquelle un «traitement » existe : autrement dit, les critères biologiques et anatomiques autorisent l'intervention. Cette posture médicale n'est pas sans rappeler les « refus de prise en charge ${ }^{2}$ » qui sont, dans la littérature sociologique, envisagés comme des processus de discrimination touchant des publics vulnérables - et fréquemment présentés comme difficiles, ou dont la demande est discréditée : patients en demande de contraceptifs (DAVIDSON et al., 2010), inscrits à la couverture maladie universelle (CARDE, 2006), personnes âgées (HIGASHI et al., 2012), etc. Ces fins de non-recevoir, qui prennent des formes très variées, rappellent que la diversité des décisions médicales ne se réduit pas à la démarche clinique mais relève aussi de procédures de catégorisation à la fois biomédicales et sociales, voire morales (JUTEL, 2009). Notons par ailleurs qu'elles semblent ne pas avoir le même sens selon les contextes : ainsi, parmi les chirurgiens plastiques, alors que le refus de prise en charge peut être loué comme un signe de professionnalisme en chirurgie esthétique, il est considéré comme un refus de soin en chirurgie réparatrice. Quelles sont les conditions permettant à un professionnel de santé de revendiquer une non-prise en charge ? Dans ce cadre, nous analysons les principes de justification mobilisés dans ces situations de désaccord et, plus avant, les enjeux professionnels qu'ils sous-tendent, notamment quant au mandat ${ }^{3}$. La consultation est, pour cela, considérée comme une épreuve, un moment au cours duquel il faut déterminer l'état des choses, mais également en rendre compte. En se centrant essentiellement sur les discours des praticiens enquêtés (voir encadré), notre article analyse moins les ressorts de l'interaction médecin-client que la façon dont les chirurgiens les rapportent. Les refus sont ainsi envisagés non seulement comme des révélateurs d'une manière de penser et d'exercer la médecine dans le cadre d'une pratique décriée, mais aussi et surtout comme une manière de légitimer une appartenance médicale. Nous formulons l'hypothèse que ces refus, que les chirurgiens évoquent à de nombreuses reprises alors qu'ils semblent en réalité peu fréquents en consultation, ont un double statut, moral et professionnel. Dans une pratique $^{4}$ parfois déconsidérée au sein de la sphère médicale et dont la structuration est particulière (cf. infra), les postures de refus constitueraient pour les chirurgiens un dispositif de revendication à la fois de leur identité de médecin et de leur autonomie professionnelle. Elles leur permettent notamment de témoigner de leur pouvoir de

2. L'expression « refus de soin », fréquemment utilisée, n'apparaît pas indiquée ici : d'une part car, dans le contexte de la chirurgie esthétique, la notion de soin est controversée (EDMONDS, 2013) ; et, d'autre part, car dans la littérature, ces fins de non-recevoir ne portent pas nécessairement sur un soin, au sens classique du terme, comme pour la non-délivrance de contraceptifs. En ne présupposant pas la nature ou l'objet du refus, nous nous interrogeons sur les manières dont les chirurgiens esthétiques définissent leurs refus d'opérer.

3. Le mandat correspond à cette possibilité accordée aux membres d'un groupe professionnel d'influer sur la manière dont est exercée leur activité, et à en préciser les contours. Comme le précise Everett Hughes (1996, p. 100), les professionnels qui s'en revendiquent « prétendent indiquer à la société ce qui, dans tel domaine de l'existence, est bon et juste pour l'individu et pour la société. En fait, ils définissent les catégories dans lesquelles ce domaine peut être pensé ». 4. Le terme, utilisé dans l'article, renvoie au fait que la chirurgie esthétique n'est pas une spécialité à part entière, faisant partie de la chirurgie plastique reconstructrice et esthétique (cf. infra). 


\section{ENCADRÉ}

\section{Enquêter sur les chirurgiens plastiques en France}

Le questionnement ici présenté est apparu au fil d'une enquête doctorale et a été soutenu par une approche inductive. En plus des 80 entretiens réalisés avec des chirurgiens plastiques entre 2008 et 2010, portant essentiellement sur leur activité professionnelle journalière, qui constituent la base de cet article, des observations non participantes (lors de congrès professionnels ou dans le quotidien de ces chirurgiens) ont été conduites. Notre présence a toutefois été limitée lors des consultations en raison des réticences exprimées par les praticiens libéraux, ce qui est un problème classique auquel sont confrontés les sociologues de la santé ${ }^{1}$. Une analyse de contenu des revues professionnelles a également été menée ; l'une d'entre elles, les Annales de chirurgie plastique esthétique, a fait l'objet d'un traitement systématique. Nos enquêtés, sollicités selon un principe de diversification maximale, se rapprochent des données démographiques recensées (LE BRETONLEROUVILlOIS, 2007). Âgé de 51 ans (contre 48 ans pour la recension nationale), l'enquêté type est un homme (à $85 \%$ contre $81 \%$ ), libéral (à $85 \%$ ), exerçant en Île-de-France (31\%). Enfin, ces chirurgiens apparaissent largement comme des « héritiers » : l'analyse de leur origine sociale révèle une forte prégnance des professions médicales et des cadres supérieurs chez le père (près de trois quarts des enquêtés), alors que la mère est dans près de deux cas sur trois sans profession et, dans environ un cas sur trois, enseignante ou de profession paramédicale.

1. Voir par exemple GUYARD (2002) pour le cas des consultations des gynécologues-obstétriciens.

prescription ou de non-prescription et, ce faisant, s'apparentent à des procédés de légitimation professionnelle.

Dans un premier temps, nous nous attachons à donner quelques éléments de contextualisation, en nous intéressant à ce qui déconsidère la chirurgie esthétique dans le monde médical, mais également à la manière dont ces professionnels présentent et définissent leur activité, et notamment ses finalités. Dans un deuxième temps, nous tentons d'identifier les éléments sur lesquels se basent ces chirurgiens pour refuser d'opérer : le plus souvent, ces refus concernent ceux qu'ils considèrent comme de « mauvais » clients. Dans un troisième et dernier temps, nous appréhendons ces refus comme une modalité rhétorique, que nous nommons éloge du refus, favorisant une présentation déontologique de l'activité, et constituant, par là même, une revendication d'autonomie dans la prescription. 


\section{La chirurgie esthétique, une sous-spécialité en quête de légitimité}

Avant de les analyser, il est nécessaire de décrire le paysage dans lequel les logiques présidant aux refus d'opérer s'inscrivent. En effet, mettre en avant les traits saillants de la chirurgie esthétique en tant qu'activité nous permettra de donner sens à ces refus. Pour ce faire, nous proposons de combiner des aspects objectivables et les significations que les chirurgiens allouent à leur geste opératoire.

\section{À activité particulière, relation médecin-client particulière ?}

En France, la chirurgie plastique reconstructrice et esthétique (CPRE) est une spécialité subdivisée en pôles dont les frontières administratives sont dessinées par l'assurance maladie. La chirurgie plastique reconstructrice est ainsi prise en charge, alors que la chirurgie esthétique reste aux frais du demandeur. La première s'étend sur un domaine varié, de la chirurgie de la main à la chirurgie des brûlés, et jusqu'aux diverses reconstructions après accidents. Plus réduite, la chirurgie esthétique se limite à quelques interventions, portant essentiellement sur les seins (augmentation ou diminution), les amas graisseux (liposuccion), les paupières (blépharoplastie), le ventre (plastie abdominale), le visage (lifting) ou le nez (rhinoplastie). La CPRE est l'une des spécialités les plus faibles numériquement avec 722 praticiens, soit $0,48 \%$ des médecins (LE Breton-Lerouvillois, 2009) et moins d'une douzaine de nouveaux inscrits à l'ordre des médecins chaque année. Cette discipline est à la fois très convoitée par les étudiants lors de leur internat et décriée car suspectée de ne pas correspondre aux valeurs du monde médical ${ }^{5}$. Cette ambivalence se cristallise sur la chirurgie esthétique, supposément plus rémunératrice ${ }^{6}$ tout en étant socialement moins légitime car le recours du client à une intervention relèverait du désir et non du besoin selon Nora JACOBSON (2000). Les épithètes dont le monde médical affuble régulièrement les chirurgiens pratiquant l'esthétique relaient des poncifs peu flatteurs : «techniciens de surface», « esthéticiennes », «barbiers », « manucures », « pédicures ${ }^{7}$ », etc. expriment, chacun à leur manière, la disqualification.

C'est dans ce cadre, et plus spécifiquement encore dans celui de sa relation avec le chirurgien, que l'autonomie du client s'inscrit, même si elle ne constitue pas une

5. Dans leur analyse des choix d'internat, Anne-Chantal HARDY-DubERnET et Yann FAURE (2006) montrent les hiérarchies symboliques des spécialités. En opposition par exemple à la médecine interne, la CPRE n'apparaît pas comme de la «vraie » médecine. Mais elle séduit, non seulement pour ses spécificités techniques, mais aussi pour le cadre qu'elle offre (peu de gardes, articulation aisée des différents temps sociaux, rémunération intéressante, installation facilitée dans le libéral).

6. La CPRE est en effet l'une des disciplines les plus lucratives du champ de la médecine. Alors que les bénéfices non commerciaux en 2006 s'élèvent en moyenne pour les chirurgiens - toutes spécialités confondues - à 85028 euros pour le secteur 1 et à 144053 euros pour le secteur 2, ils atteignent respectivement 106282 euros et 147606 euros pour les chirurgiens plastiques (VALLENCIEN, et al., 2008).

7. Ces propos ont été entendus lors d'observations à l'hôpital ou lors de congrès, mais nous ont également été rapportés par les chirurgiens plastiques eux-mêmes. 
caractéristique endémique de la sous-spécialité ${ }^{8}$. Le demandeur n'est en effet pas soumis à l'urgence, et encore moins à l'urgence vitale. En chirurgie esthétique, une opération peut sans difficulté être reportée ; le client choisit son praticien, définit son problème et est amené à débourser plusieurs milliers d'euros. La définition des objectifs opératoires renvoie en outre à un champ - la beauté - pour lequel la médecine n'a pas de monopole. Un tel contexte a conduit certains sociologues nord-américains à considérer cette activité à travers le prisme de la marchandisation, en opposition à l'activité médicale (Mirivel, 2007 ; Sullivan, 2004). Enfin, si la structuration du système d'information en médecine, partiellement régulée par le Conseil national de l'ordre des médecins, se rapproche de celle de l' « économie de la qualité », la chirurgie esthétique s'en distingue au contraire. Lucien KARPIK (1995) définit l'économie de la qualité à partir de l'opacité du marché, entretenue par la prohibition de la publicité personnelle, l'indétermination du produit et le différé de l'évaluation ; ce faisant, elle se distingue de l'économie de marché basée sur la concurrence. Or en chirurgie esthétique, l'expression publique, dans les médias, s'est imposée depuis maintenant plusieurs années ; les frontières de l'autorisé se sont élargies pour aboutir à une véritable publicité voilée, en particulier avec l'utilisation désormais massive d'internet. D'après nos calculs, plus d'un praticien sur deux dispose d'un site personnel, sur lequel le client a tout loisir de se renseigner, ou même de poser directement des questions au professionnel. Tous ces éléments sont caractéristiques de la chirurgie esthétique, qui est en cela bien différente de la chirurgie réparatrice par exemple, que de nombreux chirurgiens pratiquent parallèlement.

\section{Le levier «psy » comme justification de l'acte opératoire}

Nous l'avons évoqué, les chirurgiens pratiquant l'esthétique sont fréquemment soupçonnés de procéder à des opérations futiles, alors même qu'ils revendiquent une action thérapeutique. Selon ces praticiens, l'acte chirurgical ne viserait ainsi pas seulement à supprimer une disgrâce, mais aussi - et surtout - à libérer le client d'un mal-être, comme l'indiquent les deux chirurgiens cités ci-après :

«Le bénéfice réel de ce type de chirurgie n'est pas fonctionnel mais subjectif [...].

On conçoit aisément que si chacun peut parfaitement vivre avec des poches sous les yeux, des bajoues, ou une bosse ostéo-cartilagineuse, la demande est en fait d'origine psychique. »

(MÉNINGAUd, TouRÉ, 2005, p. 645.)

Les références que font les chirurgiens aux « effets secondaires » ou à «l'estime de soi » sont nombreuses, et renvoient aux objets de la psychothérapie. Leur faible

\footnotetext{
8. Les analyses de la relation médecin-patient constituent un champ largement travaillé par la sociologie de la santé depuis les travaux de Talcott Parsons. Si aujourd'hui la plupart des analyses reconnaissent une autonomie croissante du patient, elle apparaît de manière divergente selon les auteurs, mais aussi et surtout selon les terrains investis. Comme le soulignent les travaux classiques d'Eliot FREIDSON (1984), la structuration de chaque spécialité influe, entre autres, sur le rapport aux patients, engageant à une « soumission » plus ou moins importante.
} 
formalisation correspond toutefois à un bricolage individuel, mêlant références psychanalytiques ( «transfert»), indications psychiatriques ( dysmorphophobie ») et concepts psychologiques ( « schéma corporel », « confiance en soi », « estime de soi », etc.). Le travail sur ces aspects demeure informel, et les collaborations effectives avec des psychothérapeutes sont tout aussi rares. Dans les articles parus dans les revues professionnelles des chirurgiens plastiques, très peu de références aux travaux d'autres spécialités sont observables, et leurs sources font bien davantage appel à un cadre auto-référencé sur leur propre pratique. Les chirurgiens enquêtés insistent sur la gestion totale de leur consultation, dont les dimensions psychologiques seraient garanties par leur bon sens et leur expérience. François K. ${ }^{9}$ (libéral, quinquagénaire) évoque cette capacité à « sentir », et « l'intuition » acquise « après des années de psychologie appliquée au corps ». Certains chirurgiens revendiquent un intérêt personnel pour l'autre, ou parfois des qualités plus instinctives, « parce qu'on a ça dans nos tripes. Si on a bien choisi notre métier, on a ça dedans. On a envie de faire ça » (Erwan R., libéral quadragénaire). L'autonomie dans la conduite de la consultation, tout en mettant en avant l'identité de soignant, permet de tenir à distance les professionnels du monde de la psyché, malgré les emprunts à leur terminologie.

En raison de l'implication «psychologique » de la demande, le diagnostic est en outre présenté comme délicat. La difficulté de prévoir les réactions du client après l'opération, présentée comme un lieu commun, serait ainsi inhérente à cette relation. C'est pourquoi, pendant la consultation, ils tentent de les anticiper. Et ce d'autant plus que l'amélioration esthétique, du point de vue du chirurgien, ne s'accompagne pas nécessairement symétriquement de la satisfaction du client :

« Nous avons tous eu, dans nos consultations, des patients dont le résultat opératoire nous a déçus, mais qui étaient paradoxalement très heureux de leur intervention. Réciproquement, nous avons vu des patients, pour qui nous avions techniquement honoré le contrat, s'avérer très insatisfaits. »

(MÉningaud, Touré, 2005, p. 646.)

Les chirurgiens envisagent donc le conflit comme potentiel, et la conduite du client mécontent comme mystérieuse, incompréhensible, voire irrationnelle. Les discours que nous ont rapportés les chirurgiens ont valeur d'avertissement, les incitant à développer une posture d'écoute qui s'impose donc comme une injonction professionnelle :

«Celles qui sont pas contentes [de leur résultat], c'est qu'on s'est trompé avant $[\ldots]$ : soit c'est un patient qui ne devrait jamais se faire opérer, parce que c'est un patient qui sera éternellement insatisfait ; soit il y a un problème de dialogue, de communication. »

(Julien T., libéral, trentenaire.)

La vigilance est donc de mise afin de sonder la demande, et la consultation doit pouvoir aboutir à un refus d'opérer.

9. Afin de conserver leur anonymat, les prénoms et initiales des enquêtés ont été modifiés. 


\section{Refuser : quelques indicateurs diagnostiques}

Identifions à présent les critères que les enquêtés mettent en avant pour circonscrire les demandes problématiques - et par extension les clients problématiques -, en nous intéressant particulièrement à celles qui sont difficilement justifiables d'un point de vue biomédical.

\section{Deux figures de « mauvais » clients}

Sonder l'environnement psychosocial de la demande est présenté comme une nécessité justifiée par des récits de mésaventures largement relayés dans ce monde professionnel. Non décelées, les «mauvaises » demandes peuvent entraîner des difficultés relationnelles postopératoires : harcèlement, publicité négative, voire procès. Pour s'en prémunir, les chirurgiens affirment appuyer leur décision, et donc leur (non) prescription sur une analyse des dimensions psychiques mais aussi sociales à l'origine de la demande d'intervention. Au « mauvais » client, qu'il faut refuser d'opérer, sont ainsi fréquemment associés un étiquetage psychopathologique ou, au moins, des difficultés psychosociales. La demande de modification esthétique serait alors révélatrice de troubles comportementaux, qui prennent la forme de deux figures : la femme perdue et la femme délaissée ${ }^{10}$. Sans épuiser complètement les différents avatars de «mauvais » clients, elles en donnent toutefois un large aperçu. Dans les deux cas, la modification corporelle ne serait pas effectuée pour soi mais serait instrumentalisée : elle serait perçue comme un moyen d'accéder à un statut social, de se rapprocher de l'être aimé, ou de surpasser une souffrance affective.

$\mathrm{La}$ « bimbo », pour ces chirurgiens, incarne la figure de la femme perdue. Elle se présente sous les traits d'une jeune personne dont la quête démesurée de beauté est investie d'espoirs professionnels et sociaux. Dans ces lectures psychanalysantes, la souffrance se manifeste dans les aléas d'une existence malheureuse.

«Vous prenez une bimbo : une jeune fille plutôt jolie, qui a 20 ans, plus ou moins aimée [lorsqu'elle était] enfant, et qui est plus ou moins en souffrance psychologique. Il ne faut pas qu'elle se déforme chirurgicalement pour y arriver. Le fait de gratter un peu dans son histoire nous permet de savoir où sont ses erreurs et d'adapter son comportement [en tant que chirurgien]. »

(Marc A., libéral, quadragénaire.)

Les chirurgiens perçoivent le raisonnement de ces clientes comme tronqué, inaccessible à la rationalité, et s'inscrivent ainsi dans une tendance médicale qui qualifie les problèmes féminins de mentaux (MoscuCCI, 1993). À la distance genrée s'ajoute ici

10. Pour la dimension genrée de ce monde social, qui tout en influant sur ce jeu de catégorisations n'est pas le cœur de cet article, voir LE HÉNAFF (2013). On peut simplement évoquer la répartition par sexe des chirurgiens et patient.e.s : les femmes représentent environ $90 \%$ de la clientèle (KNIPPER, JAUFFRET, 2003) et les hommes $81 \%$ des chirurgiens (LE BRETON-LEROUVILLOIS, 2007). 
une distance sociale, ces demandeuses étant généralement désignées comme issues des catégories populaires. Dépréciée pour son instabilité et sa versatilité, cette clientèle est présentée comme étant à éviter, quitte parfois à mettre en œuvre des mesures préventives qui touchent alors les classes les plus défavorisées de leur clientèle. Philippe M. (libéral, trentenaire) fixe ainsi des honoraires élevés afin d'échapper à cette population, qu'il soupçonne d'être plus difficile à gérer.

Une autre figure soulève la méfiance : la femme délaissée. Selon les chirurgiens, elle chercherait, par le biais d'une amélioration esthétique, à consolider sa relation conjugale, voire à « trivialement garder son mec » (Brice R., libéral, quinquagénaire). La dépendance affective générerait une absence de discernement. L'article de Françoise VANDENBUSSCHE (1994, p. 522), chirurgien plastique, identifie ainsi, à propos du lifting, une catégorie problématique : « la demande précoce en France [de] la femme jeune de 30 à 35 ans qui, en phase paradépressive menstruelle, conjugale ou professionnelle, commence à se regarder et constate qu'elle n'a plus 20 ans ». Les chirurgiens expliquent faire preuve d'une vigilance toute particulière à propos des périodes difficiles traversées par ces clientes, notamment lorsqu'il s'agit de ruptures sentimentales, «genre la maman qui est en cours de divorce et qui veut un lifting parce qu'elle croit que ça ira mieux » (Claire B., professeur des universités - praticien hospitalier [PU-PH], quadragénaire). Dans pareil cas, VANDENBUSSCHE (1994, p. 522) conseille de « "surseoir" jusqu'à stabilisation événementielle ou distanciation "psychologique" par rapport à la période conflictuelle ou à la conduite de deuil... faute de quoi, l'insatisfaction éventuelle quant au geste chirurgical réalisé peut avoir un rôle d'exutoire/culpabilité par rapport au conflit vécu ». Sources de souffrance, la rupture et plus largement les difficultés familiales et affectives peuvent être considérées par les chirurgiens comme des contre-indications à l'opération. Ces postures rappellent en outre l'importance supposée de l'affect que ce groupe largement masculin attribue aux femmes.

Ces deux figures révèlent en creux les contours de ce qu'ils estiment être la «bonne » clientèle, telle qu'elle est définie dans ce monde social, et de ce qu'ils présentent comme le « vrai » travail (BECKER, 1985). Parce qu'ils répètent sans cesse que se faire opérer pour plaire à quelqu'un d'autre et pas uniquement pour soi n'est pas une bonne chose, et parce qu'ils associent systématiquement cette démarche à leur clientèle féminine, ces spécialistes instruisent l'idée d'une féminité sous influence et témoignent d'une culture chirurgicale empreinte de virilisme (ZOLESIO, 2012). En dénonçant la « bimbo », ces chirurgiens révèlent une pratique de classe, dans un monde où « avoir une belle clientèle », c'est-à-dire une clientèle solvable, est un signe de réussite et de prestige professionnel. En soulignant la multiplication des retards ou des entailles aux règles de bienséance de cette catégorie de clientes, c'est à une véritable critique sociale, qu'ils relient parfois à une « démocratisation » de la clientèle, que se livrent certains de ces chirurgiens :

«Les gens qui font le plus de procès sont les gens qui payent le moins cher. Ce sont les gens qui n'ont pas d'argent, qui payent pas, qui vont attaquer, tandis que ceux 
qui payent très cher n'attaquent jamais. C'est pas le même raisonnement dans ces clientèles. »

(Virginie S., libérale, trentenaire.)

Les manifestations en matière de mauvais goût esthétique sont d'ailleurs associées aux classes les moins privilégiées. Pour ces praticiens, la situation de ces clientes dans l'espace social est lisible en filigrane : ce sont des « filles paumées », à l'enfance « difficile », qui «n' [ont] pas toujours été aidée[s] dans la vie ». La vulgarité caractérise leur demande aussi bien que leur façon de s'habiller ou de se comporter. « Elles manquent de tenue », affirme Vincent T. (libéral, quadragénaire) : dans son appréciation, elles ne savent ni se tenir, ni se contenir. Annie G. (PU-PH, sexagénaire) dénonce « des seins comme ça, qui débordent du soutien-gorge partout ». Le corps, qui n'est plus discipliné, attire l'œil, voire suggère le sexuel. Il est « en excès », caractérisé par le « trop », quand, au contraire, ces praticiens revendiquent le « naturel ».

Instabilité psychologique et vulgarité présentée comme socialement située constituent donc deux registres de refus.

\section{Le sensible comme appui diagnostique : quand « le courant ne passe pas »}

Si la possibilité du refus est fréquemment évoquée, lors d'entretiens sociologiques ou d'interviews journalistiques, les praticiens ont parfois du mal à identifier formellement des indicateurs pour la circonscrire. Sans repère clinique, ni élément tangible ou explicité renvoyant à des figures de « mauvais » clients, certains refus s'apparentent à un pouvoir discrétionnaire qu'il apparaît compliqué de justifier. Le trouble que ces praticiens affirment éprouver est socialement significatif : le refus d'opérer conduit à une rupture du cadre interactionnel, car il constitue une « offense fortuite » (GoFFMAN, 1973, p. 173). Pour sauver la face, ils donnent le sentiment d'octroyer du crédit à la demande, faisant alors comme si « quelque chose avait été fait » (BROOM et al., p. 82). Le pieux mensonge est présenté comme le meilleur compromis pour sauver l'interaction tout en évitant d'opérer un « mauvais » client. Des programmes opératoires surchargés, une brusque augmentation des honoraires, un manque de formation à cette technique : toute excuse, au sens goffmanien du terme, est saisie pour littéralement se « débarrasser » de ces indésirables.

Les chirurgiens avancent ce qu'ils appellent le « subjectif » et le sensible comme modalités d'évaluation du client. Ils les mobilisent lorsqu'ils évoquent le malaise presque imperceptible que leur inspirent certaines interactions :

« Si j'ai un truc à l'estomac - un petit nœud - ou si je sens mes poils sur les bras qui se dressent. Je ne vais pas opérer la personne. »

(Lois L., libéral, quinquagénaire.)

Sans autre forme de justification, Édouard G. (libéral, quinquagénaire), fataliste, annonce au client que « [l'intervention] ne marchera pas ». Ces chirurgiens, au capital langagier pourtant important, sont ainsi confrontés à une indicible évidence. Cette 
« irrationalité » (dixit Lois L.) affichée interroge, dans un contexte où se multiplient référentiels et codes de bonnes pratiques.

Dans la littérature professionnelle, quelques très rares articles avancent des pistes pour alerter le praticien, comme ceux de Jean-Pierre ChAVoIN (2003) et de Françoise VANDENBUSSCHE (1994), qui dressent la liste des comportements équivoques comme le ressentiment, la froideur, l'agressivité ou la directivité.

« Il y a aussi le patient qui se présente sans chaleur, sans sourire et qui d'emblée critique son médecin de famille ou un premier chirurgien plasticien ou un autre chirurgien : "Le docteur $\mathrm{X}$ est un incapable, le docteur Y qui m'a opéré m'a complètement raté." Les exigences sont précises, les choix techniques sont imposés. Même si l'indication est valable, il faut parfois refuser pour éviter des suites désagréables et des rapports conflictuels. »

(Chavoin, 2003, p. 275.)

«Il faut privilégier la notion de bonne entente préalable avec son patient. Si un individu donné vous semble d'emblée opposant ou agressif, ou vous insupporte subjectivement... cela mérite réflexion avant contrat, voire une "distanciation" ou même un refus circonstancié et poli. L'âge et l'expérience aidant, je préfère les refuser d'emblée, quand je ne les "sens" pas, plutôt que de les subir après. »

(VANDENBUSSCHE, 1994, p. 522.)

La qualité de la relation entre le chirurgien et le client émerge largement de ces discours, au point qu'elle apparaît comme une variable de la réussite opératoire ${ }^{11}$ :

« Je vais sûrement pas opérer quelqu'un où je sens que le courant ne passe pas [...]. Je me souviens d'une dame [...]. J'avais expliqué que oui, je pouvais opérer, mais que non, je n'opérerais pas. Parce que je la sentais pas. C'était vraiment une histoire de courant qui passait pas. Et je sentais vraiment que le relationnel avec cette patiente allait être extrêmement compliqué. Elle n'a pas compris pourquoi je ne voulais pas l'opérer. Elle est partie furieuse. »

(Jérôme D., libéral, trentenaire.)

Certains affirment même, comme Marc A. (quadragénaire, libéral), et de manière plus lapidaire encore, ne pas « opérer les gens qu' [ils] n'aime[nt] pas », sans pouvoir, malgré nos relances, exprimer autre chose que ce ressentiment, cette antipathie rédhibitoire provoquée par un comportement inadapté, mais difficile à caractériser. La part de non-mesurable, ou de non-« objectivable », s'exprime sans fard. Pour autant, cette posture ne peut être considérée comme arbitraire, mais plutôt comme implicitement rationnelle et peu facilement dicible (Meershoek et al., 2007). Dans la lignée des analyses de Laurence TAIN (2005), nous faisons l'hypothèse que cette valorisation de la

11. JACOBSON (2000), à propos des plasticiens américains, relève sensiblement les mêmes formulations, évoquant même le caractère sublimatoire de cette relation. Dans la littérature, les difficultés à interagir avec certains patients en raison de leur non-observance d'un traitement, de leurs exigences, de leur volonté de multiplier les avis ou de leur hostilité ont déjà été mises en évidence (BREEZE, REPPER, 2002 ; GREENFIELD et al., 2012). Cela ne remet pour autant pas en cause leur prise en charge. 
communication par les enquêtés dissimule des processus de discrimination fondée sur l'altérisation d'une catégorie de clients présentée comme « mauvaise » ou « difficile », ce qui leur permet d'écarter avant tout les franges les plus populaires de la clientèle.

\section{La perception du risque comme indicateur de mauvaise demande}

Ces refus, qui s'appuient en partie sur le manque de « fiabilité », de « bonne entente » ou de « confiance » que le chirurgien ressent vis-à-vis de son client, révèlent, paradoxalement, sa vulnérabilité au sein de cette relation. Ces fins de non-recevoir sont en effet fréquemment associées à une anticipation des problèmes que pourrait causer le demandeur et cela, indépendamment du résultat de l'opération :

« Je vais pas opérer quelqu'un qui va pas être quelqu'un... de fiable. Les gens me disent : "Vous êtes bien !" Mais non, je suis pas bien, je suis pragmatique. Si c'est pour que la personne vienne quelques mois plus tard pour me dire, vous m' avez ratée,

j' ai pas envie de me faire emmerder. Si ça colle pas, j' ai tout intérêt à leur dire niet. »

(Erwan R., libéral, quinquagénaire.)

Il est significatif que la question du refus apparaisse dans les années 1980-1990, c'est-à-dire au moment même où émerge, dans les écrits professionnels, la problématique de la judiciarisation. Depuis, les préoccupations des chirurgiens esthétiques pour les décisions de justice portant sur des litiges avec des clients ont envahi l'espace de discussion $^{12}$, participant à la perception d'une judiciarisation rampante de leur activité. Plus que la réalité de ces risques ${ }^{13}$, c'est l'importance qui leur est accordée qui importe ici : tous les chirurgiens enquêtés ou presque y font référence comme un élément incontournable de leur pratique.

Parallèlement, ces discours alimentent l'idée d'un changement de la clientèle (HERZLICH et al., 1993) que l'augmentation perçue du risque viendrait illustrer. Une frange de ces demandeurs serait désormais plus consumériste, agressive et prompte à la dénonciation. Tous les chirurgiens plastiques enquêtés ou presque, y compris les plus jeunes, signalent cette évolution qu'ils associent à la perte de prestige de leur statut et parfois, plus largement, à des changements sociétaux. Cette référence au passé cristallise la perception d'une identité menacée, participe d'un sentiment de crise plus ou moins aigu (BERCOT, MATHIEU-FRITZ, 2007) et renforce leur sentiment d'insécurité.

12. Ce climat de méfiance est largement entretenu par les nombreuses actions mises en place par le syndicat des chirurgiens plastiques et par leur principal assureur, la SAS Branchet. La plupart des rassemblements professionnels accordent ainsi une ou plusieurs sessions à cette question, et les articles sur le sujet sont nombreux dans les revues professionnelles. Ces aspects ont également été intégrés au diplôme préparant au titre de chirurgien plastique, qui lui attribue un module entier sur les huit de la formation.

13. Une recrudescence significative de la sinistralité (nombre de déclarations d'accident corporel) est toutefois à noter : entre 1995 et 2001, elle passe en chirurgie plastique de $12,1 \%$ à 21,1 \% selon le Sou médical, société d'assurance spécialisée dans les professions médicales. Et la chirurgie esthétique est visiblement la pratique chirurgicale la plus sanctionnée (e-mémoires de l'Académie nationale de chirurgie, 2003). Selon Branchet (2003), principal courtier des plasticiens libéraux, ceux-ci doivent s'attendre à une mise en cause tous les quatre ans environ. Mais seule une sur quatre aboutit à une indemnisation. 
La fin annoncée des rites statutaires ${ }^{14}$ est mise en avant comme un symptôme de cette crise. Ces discours stigmatisent le manque de déférence qui tendrait - entre autres - à brouiller la distribution des rôles et des prérogatives :

« Je fais en sorte d'éviter les personnes pas fiables, des patients qui n'ont pas une conception équivalente de ce que j'attends d'un patient. Si au bout de trois consultations, vous voyez qu'il n'a rien compris et que de toute façon il ne veut pas chercher à comprendre, qu'il a vu que la voisine avait été opérée comme ça. Ceux aussi qui ne sont pas très respectueux de vous-même. Ils sont en retard, ils oublient un rendezvous. [...] Il ne faut pas chercher à opérer, dans le domaine de la chirurgie esthétique, des patients qui n'ont pas un minimum d'affinités avec vous. »

(Tristan L., libéral, quinquagénaire.)

Les chirurgiens enquêtés indiquent que ces clients ne leur inspirent aucune sympathie en raison de la succession d'offenses qu'ils ont commises à leur égard, à la fois en tant qu'individu, mais également et surtout, en tant que médecin : ils considèrent leur attitude comme annonciatrice de contentieux à venir. Un tel manque de déférence à leur égard est présenté comme une remise en question profonde du rôle qu'ils s'attribuent et qui cristallise leurs revendications et attentes.

Pour ces praticiens dont l'activité est considérée comme moins « médicale », ce rôle ${ }^{15}$ de médecin est appréhendé comme un rempart auquel ils peuvent s'identifier et référer leurs conduites ; il leur permet aussi de revendiquer une certaine emprise dans la gestion de la consultation. En creux, ils attestent de leurs difficultés à imposer leur expertise. Ces professionnels de santé ressemblent à ceux décrits par Geva GREENFIELD et ses coauteurs (2012) qui, en situation critique, oscillent entre sentiment de vulnérabilité et revendication à l'autonomie professionnelle. Une partie de l'activité des chirurgiens enquêtés semble pouvoir être traitée sous cet angle d'analyse ${ }^{16}$. La revendication du refus apparaît de fait liée au travail de négociation des rôles de chacun. Les registres argumentaires pointent des revendications professionnelles à l'autonomie, soutenues par une approche présentée comme une éthique du diagnostic selon laquelle refuser s'oppose à un démarchage clientéliste. Sans user nécessairement du refus, Gwendoline T. revendique ce travail de définition de son domaine d'action quand elle use sciemment de stratagèmes à même de recadrer le client trop peu respectueux de la dimension médicale de la consultation : elle se focalise par exemple sur les antécédents médicaux, sans s'enquérir des motifs pour lesquels il vient consulter, inversant de la sorte la chronologie classique de la consultation. La déstabilisation du client - but recherché de ce procédé - vise à décentrer l'individu de sa disgrâce, afin de réaffirmer

14. Démonstration du pouvoir accordé au médecin, ils consistent par exemple à ce que les médecins soient appelés « docteur ».

15. Le concept de rôle est un pivot intéressant pour l'analyse. D'abord car il permet de rappeler les structures sociales à l'œuvre, mais aussi car les individus eux-mêmes y font référence, et le composent, laissant entrevoir non pas un modèle strict de comportements, mais un arrangement de modèles.

16. En ce sens, leur activité n'est pas sans présenter quelques analogies avec l'analyse que propose BECKER (1985) sur les policiers : l'une de leurs préoccupations est justement d'être traités avec la déférence qu'ils estiment due à leur rôle, invitant à un travail de figuration, tel que l'entend GofFMAN (1973). 
l'importance des questions plus strictement médicales. Ce faisant, le praticien a pour ambition de maîtriser la situation et de renvoyer chacun à ses attributions :

«Ils ne sont pas venus voir l'esthéticienne ou la coiffeuse, ils sont venus voir un médecin [...]. Ça déstabilise un peu les gens, et ça, je reconnais que j'en joue un petit peu. Parce que les gens viennent en disant : "Je viens pour un lifting." On leur dit : "Attendez. Là, c'est moi qui dirige la consultation, pas vous. Moi, je veux savoir ce que vous faites comme activité professionnelle, je veux savoir vos antécédents." Ça les assied un petit peu. "Vous ne venez pas que pour un lifting. Quand vous allez voir le chirurgien viscéral, vous ne venez pas pour une appendicectomie. Vous venez parce que vous avez mal au ventre. Donc on va faire dans l'autre sens. Qu'est-ce qui vous plaît pas dans votre visage ?" Et l'indication, c'est nous qui la posons, c'est pas le patient. »

En mettant en scène sa position d'expert, Gwendoline T. effectue un travail de réinscription de cette interaction dans ce qu'elle considère être une relation médicale : au client la plainte que le médecin doit examiner ; au médecin de proposer une solution ou d'éconduire la plainte. En agissant de la sorte, autrement dit en tentant d'asseoir sa décision et sa légitimité, le chirurgien s'arroge une exclusivité, celle de l'indication, sur laquelle il estime avoir toute mesure : il trace ainsi le périmètre de son autonomie professionnelle, ce qui lui permet de mettre à distance le jugement du profane. Une telle approche a également pour but d'amener le client à rapprocher ses préoccupations de celles du praticien et, dans une certaine mesure, à adopter le même regard que lui. En d'autres termes, il ne s'agit pas pour le client de solliciter seulement un technicien devant le servir (dans la dimension servile du terme), mais un professionnel auquel il abandonne une partie de sa capacité de jugement (KARPIK, 1995).

\section{Le refus, un enjeu professionnel}

Dans ces discours, le refus d'opérer est généralement associé au fait que le nécessaire lien de confiance avec le client n'a pas été établi. Corollaire du « courant qui ne passe pas », « ne pas faire confiance » est présenté comme un motif acceptable pour se dessaisir d'un client, ce qui est parfois revendiqué comme un « droit réciproque », voire un « besoin » pour Mathieu G. (libéral, quinquagénaire). Nous questionnons désormais la signification de cette notion de confiance sous deux versants : interactionnel, dès lors qu'elle se réfère aux revendications de professionnalité ; et professionnel, comme support moral et rhétorique du groupe.

\section{La confiance, une emprise interactionnelle}

Que le chirurgien revendique comme un droit le fait d'avoir confiance en son client est d'autant plus singulier que la littérature visant à éclairer les relations clientmédecin s'est presque exclusivement consacrée à la confiance que le client accorde à 
son médecin ${ }^{17}$ (Mechanic, MeYer, 2000). Si, de fait, elle ne recouvre pas les mêmes dimensions selon les acteurs (le demandeur expose son corps et sa santé), la confiance résulte en premier lieu d'un jugement porté sur une situation à risque ou perçue comme telle (GIDDENS, 1994). Que des professionnels de santé comme nos enquêtés mobilisent cette notion révèle à la fois leur vulnérabilité et leur perception du client comme risque potentiel : interactionnel, juridique mais aussi professionnel. Le lien apparaît ainsi étroit entre le manque de reconnaissance estimé de leur professionnalité et le risque perçu que la notion de confiance vient ici exprimer. Le besoin de confiance met dès lors en exergue leurs difficultés à asseoir la légitimité de leur expertise.

Pour ces chirurgiens, la confiance se construit sur deux grands principes. Le premier, mis en avant précédemment, insiste sur la reconnaissance par le client de leur position d'expert : client et médecin sont alors au clair sur ce qui relève du travail du médecin d'une part, et du souhait du client d'autre part. L'agacement dont font preuve les praticiens enquêtés face aux clients qui, avec trop d'insistance, évoquent des techniques qu'eux-mêmes récusent est significative. Qui plus est, ces professionnels exigent l'incarnation de cette confiance : le client ne doit pas seulement avoir foi en la technologie médicale, mais également en l'individu qui la porte. Pour reprendre les termes d'Anthony GIDDENS (1994, p. 35), au-delà du système expert, autrement dit au-delà des «domaines techniques ou de savoir-faire professionnel », le client doit témoigner sa confiance au chirurgien en tant que point d'accès à ce système expert. Plusieurs enquêtés affirment par exemple que les prix élevés qu'ils affichent les prémunissent d'une clientèle uniquement attirée par des tarifs bas ${ }^{18}$. Les honoraires, mais aussi la proximité géographique ou encore le hasard sont considérés comme des critères de choix peu recevables. De la même manière, la plupart de ces praticiens préfèrent qu'un client leur ait été directement adressé, sur les conseils d'un proche ou d'un confrère : les prémices de la confiance sont alors sur un terrain plus favorable ${ }^{19}$. D'autres, certes peu nombreux, encouragent même leurs clients à prendre différents avis ou, du moins, n'écartent pas cette possibilité, justement car elle permettrait de renforcer la confiance. Les pratiques de ce groupe professionnel vont ainsi à l'encontre du raisonnement économique néoclassique selon lequel la décision repose essentiellement sur les évaluations tarifaires et une standardisation du service fourni. En présentant leur geste comme unique, non mesurable, et l'indication comme individualisée, ces chirurgiens mettent en scène leur professionnalité et récusent toute standardisation des actes relevant de leur spécialité. Le profane est ainsi mis à distance, la connaissance étant marquée par la complexité des éléments à prendre en compte :

17. À quelques rares exceptions près, dont MEMBRADO (2014).

18. Faire le choix d'honoraires peu élevés est fréquemment présenté comme un risque : celui d'attirer une clientèle séduite par l'attractivité du tarif - et donc « recrutée » dans les franges socialement moins favorisées - alors que ce recrutement devrait s'appuyer sur la réputation et la confiance envers le chirurgien. Ces discours constituent un dispositif argumentatif justifiant une grille tarifaire élevée.

19. En outre, le médecin correspondant, en orientant le patient, favorise un discours homogène et concordant (CASTEL, 2005). 
« Ils voient souvent deux trois chirurgiens avant de se faire opérer. Et ça, ça ne me gêne pas [...]. Je suis certain que même lorsque tout va bien, on peut trouver des choses à dire ou à améliorer. Je veux que cette marge de, non pas d'erreur, mais d'appréciation, soit en quelque sorte gommée par la bonne relation que nous avons. Je suis sûr qu'un patient chiant, ou avec lequel je suis chiant, devant un petit truc pourrait faire tout un foin. Alors que quand on a une bonne relation, je suis sûr qu'il y a beaucoup de choses qui peuvent passer [...]. Ça facilite la discussion ultérieure. Et justement, je trie, en quelque sorte, sur la qualité de la relation que je peux avoir avec eux. »

(Romain C., libéral, trentenaire.)

Si le choix du praticien a tant d'importance pour Romain C., c'est que la personnalisation de la relation et la confiance qui en découle lui permettent plus aisément de définir le résultat, dans un domaine qui, comme l'esthétique, comporte une large part de subjectivité. Le client doit ainsi faire la preuve de son adhésion à la démarche du praticien via ce que ces médecins appellent « confiance » et qui s'apparente à une forme de loyauté. Si la réputation du praticien avant même le premier rendez-vous, sur laquelle s'appuie une confiance préalable, permet dans un premier temps d'attirer le client, celle qui s'installe au fil de la relation aide le chirurgien à définir sereinement les résultats attendus, voire à gérer les résultats indésirables. La reconnaissance de la professionnalité du praticien par le client implique que celui-ci accepte de déléguer partiellement son pouvoir de juger. Romain C. se délecte d'ailleurs de ces moments où le client, gêné, s'excuse de venir lui demander quelques explications sur la persistance d'une disgrâce qui pourrait lui être imputée en tant que chirurgien : "Y'en a même qui viennent s'excuser de m'embêter de ce qui, à l'origine, est une erreur technique de ma part. Ça, c'est génial. » Cette confiance offre également et vraisemblablement une emprise sur la trajectoire du client qui, insatisfait, constitue un risque en puissance ${ }^{20}$. La dépendance du client à son chirurgien donne de la latitude d'action à ce dernier qui aspire à influer sur la manière dont le demandeur jauge, en amont, le problème esthétique et, en aval, la transformation corporelle. En d'autres termes, cette posture vise à renforcer les logiques de régulations professionnelles : sous l'emprise d'une autorité de type charismatique et engagés dans une relation de prise en charge personnalisée, les clients délèguent à leur chirurgien leur pouvoir de contrôle.

\footnotetext{
20. Et cette crainte n'est pas sans fondement : Jean-Paul MÉNINGAUd (1999), dans une recherche portant sur vingt plaintes, montre qu'une partie de celles-ci est liée au fait que les patients se soient à un moment donné détournés du praticien poursuivi. Cette attitude corrobore un autre leitmotiv de la profession, l'injonction à garder le contact avec le patient. Cette posture a pour effet de déculpabiliser le chirurgien auquel il est fréquemment indiqué dans la littérature professionnelle que tous sont confrontés à des difficultés postopératoires et que seule la gestion interindividuelle permet d'éviter la poursuite judiciaire : «Le praticien qui n'est pas attaqué, c'est celui qui sait gérer la complication » (RoFFÉ et al., 2004, p. 641).
} 


\section{Une rhétorique professionnelle : l'éloge du refus}

Pour conclure, nous proposons de considérer les refus d'opérer dans une perspective rhétorique que nous nommons éloge du refus. Les praticiens enquêtés consacrent aux refus une expression leitmotiv : «La bonne opération, c'est celle que l'on ne fait pas. » À la fois principe absolu et posture déontologique, une telle doctrine trace les frontières de la professionnalité et définit donc le «bon » chirurgien (et par extension le « bon » client) ; au-delà de l'autonomie professionnelle revendiquée, elle aurait donc vocation à influencer la conduite des praticiens mais aussi l'image qu'ils donnent de leur activité. L'éloge du refus participe en cela à la (tentative de) construction d'une figure positive du groupe des chirurgiens plastiques. En promouvant la précaution, il invite à penser que le bénéfice financier n'est pas la motivation première du chirurgien . Cet éloge du refus peut être considéré comme rhétorique en raison de la distorsion entre son énonciation (très fréquente) et le nombre de refus effectifs, somme toute rares selon nos observations, mais érigés en symbole de la probité du groupe.

Cette posture « déontologique » est en outre associée à une réussite professionnelle dans la mesure où « tout le monde ne peut pas se le permettre » (Bruno V. quinquagénaire, hospitalier). Elle attesterait d'une clientèle et d'une réputation établies :

«Quand on a une activité suffisante pour remplir son agenda toutes les semaines, on a ce luxe de pouvoir refuser. Quand on a du mal à boucler les fins de mois, on est moins difficile. »

(Jérôme D., libéral, trentenaire.)

Ces refus, synonymes d'une « belle » clientèle pour les praticiens les plus expérimentés, seraient pour les plus jeunes une manière de se la constituer selon le principe d'un cercle décrit comme vertueux. Ce groupe professionnel considère en effet que le chirurgien dispose d'une emprise forte sur les individus qui le sollicitent et qu'il participe, grâce à la sélection qu'il effectue quotidiennement, à la construction de sa clientèle : «Un chirurgien acquiert sa réputation non pas en opérant, mais en refusant d'opérer », comme le signale Jean-Luc Roffé (2006, p. 98), chirurgien plastique. En d'autres termes, le bon praticien disposerait d'une bonne clientèle grâce à un tri soigneux et à une approche non marchande de son activité : « La clientèle est à l'image du chirurgien. » La rigueur sélective, désavantageuse en termes de chiffre d'affaires à court terme, deviendrait bénéfique à long terme.

« Au bout d'un certain temps, on a la clientèle qu'on mérite quelque part. C'est-à-dire qu'un certain nombre de gens ne viennent pas ici parce qu'ils savent que je vais leur dire non. Ils savent où on va leur dire oui. »

(Vincent T., libéral, quinquagénaire.)

Cette politique de la modération s'attribue les vertus d'un exercice responsable dans un champ soupçonné d'encourager l'excès. L'éloge du refus fait en ce sens écho à l'« idéologie du désintéressement » à laquelle sont moralement et structurellement contraints les avocats que décrit Anne BoIGEOL (1981). Mais cette idéologie s'avère 
également être une ressource permettant de capitaliser une plus-value symbolique et de dissimuler des logiques plus financières. Ici, et si aucune contrainte structurelle de la sorte ne pèse, il s'agit de faire preuve d'un désintéressement financier (refuser malgré une bonne indication anatomique) tout en s'autorisant (sous couvert de déontologie) à écarter les clients que l'on juge indignes ou potentiellement dangereux. La porte est donc ouverte aux attitudes discriminatoires dont les clients hommes ont pu faire les frais (LE HÉNAFF, 2013). En confirmant la présence de jugements moraux dans la décision médicale, de telles situations renvoient aux travaux sur les refus de prise en charge. Comme nous l'avons vu, les refus rencontrés au cours de notre enquête s'appuient également sur une revendication implicite à l'autonomie professionnelle. L'éloge du refus, professionnellement construit, offre donc un dispositif argumentatif permettant de refuser une opération sur des bases au fond potentiellement discriminatoires, que les chirurgiens recouvrent d'un vernis éthique.

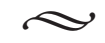

Dans cet article, nous nous sommes intéressé au cas original des refus d'opérer en chirurgie esthétique. Écartant les réserves anatomiques ou physiologiques, les chirurgiens associent leurs décisions à l'inadéquation entre le comportement ou la demande du client et leurs propres attentes. Le demandeur est alors rangé dans la catégorie du client à problèmes, en raison des conflits potentiels, voire des ennuis judiciaires, qu'il pourrait générer. Différents niveaux de lecture ont été proposés qui ont pour dénominateur commun le recours aux notions d'autonomie et d'identité professionnelles.

Ces refus montrent tout d'abord que les chirurgiens revendiquent une autonomie dans un champ, celui des corrections disgracieuses, dans lequel leur regard médical est en concurrence avec celui du client comme avec ceux de nombreux autres professionnels de la beauté et qui, par conséquent, échappe pour partie à leur expertise. Les cadres gestionnaire (non-remboursement des actes chirurgicaux) et médical (nonurgence) fragilisent l'autorité médicale du praticien face à l'émergence de clients potentiellement plus autonomes. Cette vulnérabilité semble propre à la chirurgie esthétique, puisque ces praticiens, qui exercent parfois également en chirurgie réparatrice, ne font pas mention de ce type de refus pour cette dernière. Ces fins de non-recevoir, telles qu'elles sont présentées, font écho aux travaux analysant les dispositifs que les professionnels mettent en œuvre pour garder leurs clients à distance et minimiser leur pouvoir de contrôle (MENCHIK, JiN, 2014). Par ailleurs, l'éloge du refus met en scène la professionnalité de ces chirurgiens et, en cela, participe à leur construction identitaire de médecin. Cette légitimité est recherchée par les enquêtés qui clament être sous le mandat de la médecine, celui de soigner, et revendiquent son indépendance de jugement, mais aussi son éthique : ils dénoncent ainsi les pratiques marchandes et mettent en avant une approche strictement thérapeutique qui dépasse le cadre de la recherche esthétique. 


\section{BIBLIOGRAPHIE}

BECKeR H. S., (1985), Outsiders. Études de sociologie de la déviance, traduit de l'américain par J.-P. Briand et J.-M Chapoulie, Paris, Métailié.

Bercot R., MATHIEU- FritzA. (2007), « La crise de recrutement des chirurgiens français : entre mythes et réalités », Revue française de sociologie, vol. 48, nº 4, pp. 751-779.

Boigeol A. (1981), « De l'idéologie du désintéressement chez les avocats », Sociologie du travail, vol. 23, $\mathrm{n}^{\circ} 1$, pp. 78-85.

Breeze J. A., RePPer J. (2002), “Struggling for Control: The Care Experience of 'Difficult' Patients in Mental Health Services”, Journal of Advanced Nursing, vol. 28, n 6, pp. 1301-1311.

Broom A., Broom J., Kirby E. (2014), "Cultures of Resistance? A Bourdieusian Analysis of Doctors' Antibiotic Prescribing”, Social Science \& Medicine, vol. 110, pp. 81-88.

Broussard V., Loriol M., CAROly S. (2006), « Catégorisation des usagers et rhétorique professionnelle. Le cas des policiers sur la voie publique », Sociologie du travail, vol. 48, $\mathrm{n}^{\circ} 2$, pp. 209-226.

CARDE E. (2006), « “On ne laisse mourir personne.” Les discriminations dans l'accès aux soins », Travailler, n ${ }^{\circ} 16$, pp. 57-80.

CAstel P. (2005), « Le médecin, son patient, et ses pairs », Revue française de sociologie, vol. 46, n⿳3 3, pp. 443-467.

Chavoin J.-P. (2003), « Chirurgie esthétique et éthique », Annales de chirurgie plastique esthétique, vol. 48, $\mathrm{n}^{\circ}$ 5, pp. 273-278.

Davidson L. A., Pettis C. T., Joiner A. M., Cook D. M., Klugman C. M. (2010), "Religion and Conscientious Objection: A Survey of Pharmacists'Willingness to Dispense Medications", Social Science \& Medicine, vol. 71, $\mathrm{n}^{\circ}$ 1, pp. 161-165.

Edmonds A. (2013), "Can Medicine Be Aesthetic? Disentangling Beauty and Health in Elective Surgeries”, Medical Anthropology Quarterly, vol. 27, n 2, pp. 233-252.

Freidson E. (1984), La Profession médicale, traduit de l'américain par A. Lyotard-May et C. Malamoud, Paris, Payot.

Giddens A. (1994), Les Conséquences de la modernité, traduit de l'anglais par O. Meyer, Paris, L'Harmattan.

GofFMAn E. (1973), La Mise en scène de la vie quotidienne. Volume 1. La présentation de soi, Paris, Éditions de Minuit.

Greenfield G., Pliskin J. S., Feder-Bubis P., Wientroub S., Davidovitch N. (2012), "Patientphysician Relationships in Second Opinion Encounters. The Physicians' Perspective", Social Science \& Medicine, vol. 75, $\mathrm{n}^{\circ}$ 7, pp. 1202-1212.

GuYard L. (2002), « Consultation gynécologique et gestion de l'intime », Champ psychosomatique, $\mathrm{n}^{\mathrm{o}} 27$, pp. 81-92. 
Hardy-Dubernet A.-C., Faure Y. (2006), « Le choix d'une vie... Étude sociologique des choix des étudiants de médecine à l'issue de l'examen classant national en 2005 », Document de travail, Série Études, $n^{\circ}$ 66, Drees.

Herzlich C., Bungener M., Paicheler G. (1993), Cinquante ans d'exercice de la médecine en France. Carrières et pratiques des médecins français, Paris, Inserm, Doin.

Higashi R. T., Tillack A., Steinman M., Harper M., Johnston B. (2012), "Elder Care as 'Frustrating' and 'Boring': Understanding the Persistence of Negative Attitudes Toward Older Patients among Physicians-in-Training”, Journal of Aging Studies, vol. 26, n 4, pp. 476-483.

Hughes E. C. (1996), Le Regard sociologique. Essais choisis, Paris, École des hautes études en sciences sociales.

Jacobson N. (2000), Cleavage: Technology, Controversy, and the Ironies of the Man-Made Breast, New Brunswick (NJ), Rutgers University Press.

Jutel A. (2009), "Sociology of Diagnosis: a Preliminary Review”, Sociology of Health \& Illness, vol. 31, n 2, pp. 278-299.

KARPIK L. (1995), Les Avocats, entre l'État, le public et le marché, XIII-XX ${ }^{e}$ siècle, Paris, Gallimard.

KNIPPER P., JAUFFRET J.-L. (2003), « Instantané esthétique : le questionnaire sur les interventions et leurs complications », Annales de chirurgie plastique reconstructrice esthétique, vol. 48, n 5 , pp. 299-306.

Le Breton-Lerouvillois G. (2007), L'Atlas de la démographie médicale en France. Situation au $1^{\text {er }}$ janvier 2007, Paris, Ordre national des médecins.

Le Breton-Lerouvillois G. (2009), L'Atlas de la démographie médicale en France. Situation au $1^{\text {er }}$ janvier 2009, Paris, Ordre national des médecins.

LE HÉNAFF Y. (2013), « Catégorisations professionnelles des demandes masculines de chirurgie esthétique et transformations politiques de la médecine », Sciences sociales et santé, vol. 31, $\mathrm{n}^{\circ} 3$, pp. 39-64.

Mechanic D., Meyer S. (2000), "Concepts of Trust among Patients with Serious Illness", Social Science \& Medicine, vol. 51, n ${ }^{\circ}$ 5, pp. 657-668.

Meershoek A., Krumeich A., Vos R. (2007), "Judging Without Criteria? Sickness Certification in Dutch Disability Schemes”, Sociology of Health and Illness, vol. 29, n 4, pp. 497-514.

Membrado M. (2014), « La confiance et les enjeux de la reconnaissance dans l'interaction médecin-patient en médecine générale », in Pennec S., Le Borgne-Uguen F., Douguet F. (dir.), Les Négociations du soin. Les professionnels, les malades et leurs proches, Rennes, Presses universitaires de Rennes, pp. 51-66.

Menchik D. A., Jin L. (2014), “When Do Doctors Follow Patients' Orders? Organizational Mechanisms of Physician Influence”, Social Science Research, vol. 48, pp. 171-184.

MÉNIngaud J.-P. (1999), Analyse des plaintes formulées à l'occasion de préjudices mineurs par des patients après chirurgie plastique esthétique, mémoire dans le cadre du DU «Études relatives à la réparation juridique du dommage corporel », Université Paris Descartes. 
MÉningaud J.-P., Touré G. (2005), « Recherche évaluative en chirurgie esthétique maxillofaciale », EMC-Chirurgie, vol. 2, nº 6, pp. 686-693.

Mirivel J. (2007), "Managing Poor Surgical Candidacy: Communication Problems for Plastic Surgeons", Discourse \& Communication, vol. 1, n 3, pp. 309-336.

Moscucci O. (1993), The Science of Woman: Gynaecology and Gender in England, 1800-1929, Cambridge (England), New York, Cambridge University Press.

Roffé J.-L., Porte A., Flageul G., Lacoeuilhe G. (2004), « La gestion juridique des complications en chirurgie esthétique », Annales de chirurgie plastique esthétique, vol. 49, n 6 , pp. 637-643.

Roffé J.-L. (2006), « Chirurgie esthétique : mythe et réalité », Annales de chirurgie plastique esthétique, vol. 51, n 2, pp. 97-98.

Stivers T. (2002), "Participating in Decisions about Treatment: Overt Parent Pressure for Antibiotic Medication in Pediatric Encounters", Social Science and Medicine, vol. 54, $\mathrm{n}^{\circ} 7$, pp. 1111-130.

Sullivan D. A. (2004), Cosmetic Surgery: The Cutting Edge of Commercial Medicine in America, New Brunswick (NJ), Rutgers University Press.

TAIN L. (2005), « Refus des médecins, abandons des couples : quel contrôle pour la pratique de procréation assistée ?», Sciences sociales et santé, vol. 23, n 3, pp. 5-30.

VAllencien G., Aubart F., CuQ P. (2008), Quels modes de rémunérations pour les chirurgiens? Situation actuelle et perspectives, Rapport à la demande de X. Bertrand, ministre de la Santé et des Solidarités.

VANDENBUSSCHE F. (1994), «Avant-propos », Annales de chirurgie plastique esthétique, vol. 39, n 5 , pp. 521-523.

Zolesio E. (2012), Chirurgiens au féminin ? Des femmes dans un métier d'hommes, Rennes, Presses universitaires de Rennes. 